\title{
Palpable Masses Sponsor Defined Identifier
}

National Cancer Institute

\section{Source}

National Cancer Institute. Palpable Masses Sponsor Defined Identifier. NCI Thesaurus.

Code C119915.

One or more sponsor defined characters used to identify, name, or characterize the palpable masses assessment. 